\title{
Solitary skin-colored nodule on a child's face
}

\section{Mariem Tabka, Refka Frioui, Taghrid Tlili, Nedia Fetoui, Amina Ounallah, Colandane Belajouza, Mohamed Denguezli}

Department of Dermatology, Farhat Hached Hospital of Sousse, Sousse, Tunisia

Corresponding author: Refka Frioui, MD, E-mail: Rafkouna1993@gmail.com

Sir,

A healthy, six-year-old boy presented with a slowly grown dome-shaped nodule on the mandibular angle region present for two years. The patient's past medical and family history were unremarkable. A physical examination revealed a solitary, $1.3 \times$ $1 \mathrm{~cm}$, firm, painless, flesh-colored tumor (Fig. 1). Dermoscopy showed branching, serpentine vessels on a pink background (Fig. 2a). These features disappeared when slight pressure was exerted on the dermoscope and the tumor exhibited a central, white, structureless area (Fig. 2b). An excisional biopsy was performed. A microscopic examination showed a wellcircumscribed, paucicellular dermal tumor composed of eosinophilic collagen bundles separated by clefts and forming a storiform pattern. Scattered fibroblasts were found among the collagen bundles. The overlying epidermis was slightly flattened (Fig. 3). The diagnosis of solitary sclerotic fibroma was established.

Sclerotic fibroma (SF), also known as storiform collagenoma, is a rare benign skin tumor. It usually

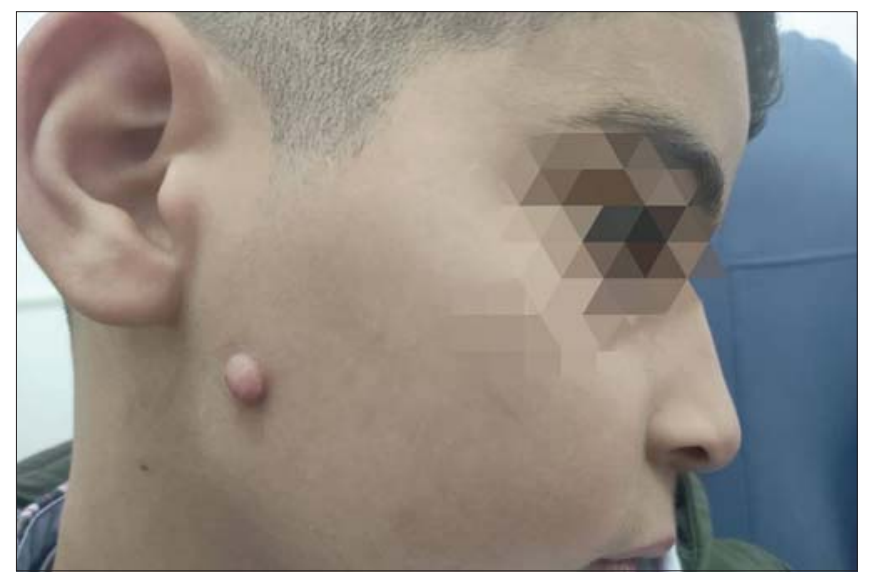

Figure 1: A solitary, $1.3 \times 1 \mathrm{~cm}$, firm, painless, flesh-colored tumor. manifests itself as an asymptomatic, slowly growing, white-to-skin-colored papule or nodule [1]. It was first described in patients with Cowden's disease, yet may also occur sporadically [2]. There were no mucocutaneous features of Cowden's disease (tricholemmomas, oral fibromas, acral keratoses, palmar pits, and gingival and palatal papules) in the patient and her family members.

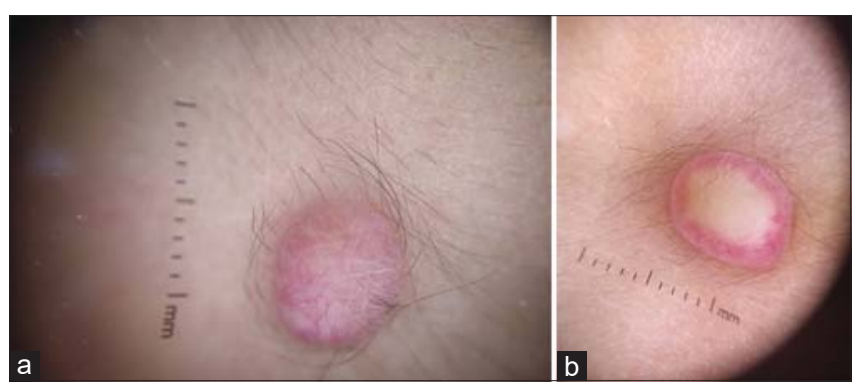

Figure 2: (a) Dermoscopy revealing branching, serpentine vessels on a pink background; (b) the tumor exhibiting a central, white, structureless area with slight pressure being exerted on the dermoscope.

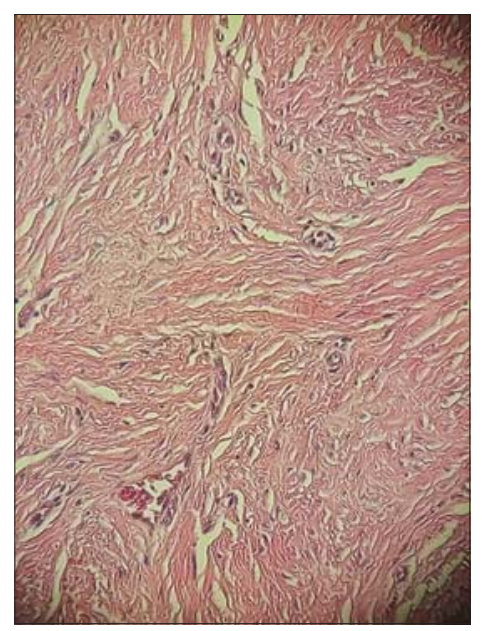

Figure 3: A well-circumscribed, paucicellular dermal tumor composed of eosinophilic collagen bundles separated by clefts and forming a storiform pattern; scattered fibroblasts present among the collagen bundles; the overlying epidermis slightly flattened.

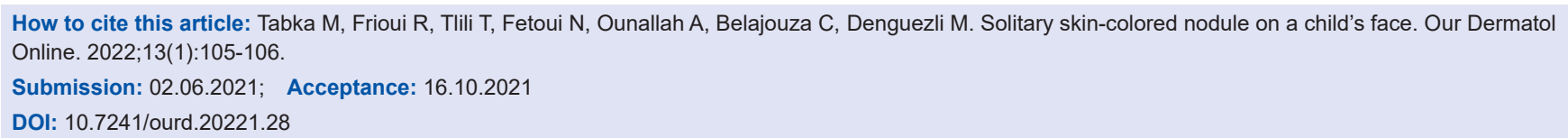


Dermatofibroma, the main differential diagnosis of SF, usually exhibits hyperplastic changes of the epidermis instead of atrophy, and the boundaries of the lesion are unclear [2]. Only two papers have been published describing the dermoscopic findings of SF, consisting of a white background with peripheral arborizing vessels [3]. A white background may be related to an increased dermal collagen density. It is also described in dermatofibroma, typically with a peripheral pigmentation network. Although dermoscopy may improve the clinical diagnosis of SF, histopathological analysis is required.

\section{Consent}

The examination of the patient was conducted according to the principles of the Declaration of Helsinki.

The authors certify that they have obtained all appropriate patient consent forms, in which the patients gave their consent for images and other clinical information to be included in the journal. The patients understand that their names and initials will not be published and due effort will be made to conceal their identity, but that anonymity cannot be guaranteed.

\section{REFERENCES}

1. Kutzner H, Lazar AJ, Patel RM, Wang WL. Fibromas. WHO Classification of Skin Tumours. $4^{\text {th }}$ ed. Lyon: IARC; 2018:314-7.

2. Tosa M, Ansai SI, Kuwahara H, Akaishi S, Ogawa R. Two cases of sclerotic fibroma of the skin that mimicked keloids clinically. J Nippon Med Sch. 2018;85:283-6.

3. Ebadian M, Citarella L, Collins D, Diaz-Cano S, Pozo-Garcia L. Dermoscopy of a solitary storiform collagenoma. Dermatol Pract Concept. 2018;8:120-2.

Copyright by Mariem Tabka, et al. This is an open access article distributed under the terms of the Creative Commons Attribution License, which permits unrestricted use, distribution, and reproduction in any medium, provided the original author and source are credited.

Source of Support: Nil, Conflict of Interest: None declared. 